\title{
Adiabatic Theory of Wannier Threshold Laws and Ionization Cross Sections
}

J. H. Macek and S. Yu. Ovchinnikov

to be published in the Proceedings of

Atomic Collisions:

A Symposium in Honor of Christopher Bottcher (1945-1993)

Oak Ridge, Tennessee

March 3-5, 1994

"The submitted manuscript has been authored by a contractor of the U.S. Government under contract No. DE-AC05-960R22464. Accordingly, the U.S. Government retains a nonexclusive, royalty-free license to publish or reproduce the published form of this contribution, or allow others to do so, for U.S. Government purposes."

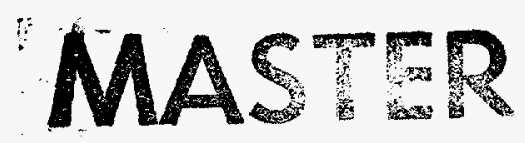




\title{
Adiabatic Theory of Wannier Threshold Laws and Ionization Cross Sections
}

\author{
J. H. Macek and S. Yu. Ovchinnikov* \\ Department of Physics and Astronomy, University of Tennessee, Knoxville, TN \\ 37996-1501 \\ and \\ Oak Ridge National Laboratory, Post Office Box 2009 \\ Oak Ridge, TN 37831
}

The Wannier threshold law for three-particle fragmentation is reviewed. By integrating the Schrödinger equation along a path where the reaction coordinate $R$ is complex, anharmonic corrections to the simple power law are obtained. These corrections are found to be non-analytic in the energy $E$, in contrast to the expected analytic dependence upon $E$.

\section{INTRODUCTION}

The collective motion of charged particles is fundamental to atomic physics. One of the most striking manifestations of this motion is the Wannier threshold law (1-8) which states that the cross section for the break up of three charged particles, where not all particles have the same sign of the charge, is proportional to $(E-I)^{\zeta W}$ where $\zeta_{W}$ is some non-integral index. For fragmentation into two electrons and a proton, this index equals 1.1269, as discovered by Wannier in his seminal paper on the subject. Wannier used a judicious combination of quantum and classical mechanics to obtain this result. One of the great challanges posed by this work, and one that intensely interested Chris Bottcher, was to fit this result into standard theories of atomic structure and dynamics (9). Progress in this direction has been incremental. Rau (2) and also Peterkop (3) were able to give a completly quantal derivation of this law, a first step towards building a general theory. The experiments of Lubell and coworkers (10) and their interpretation by Greene and Rau (11) showed that one must also include final breakup configurations with non-zero angular momentum $\mathrm{L}$ and total spin S. The Rau-Peterkop theory was extended to arbitrary three-particle systems, $(4,7)$ but none of these methods indicated how to compute the fragmentation cross section on an $a b$ intio basis. In this respect the work of Crothers (12) is a notable exception. He modified the Peterkop wave function and used it to evaluate the fragmentation matrix element. The calculation was carried out for electron impact on atomic helium 
and good agreement with the data for $E-I<2 \mathrm{eV}$ was obtained. In many respects this was a definitive result. It showed how to obtain a cross section of the Wannier form with a non-analytic coefficient $(E-I)^{\zeta W}$ multiplying an analytic function of energy $E$ with no adjustable parameters. Because the final state wave function is assumed to be of the Peterkop form, this calculation could not settle the question of whether some other form might actually emerge in a complete solution of the Schrödinger equation for this problem. To obtain some insights on this fundamental question Chris and others turned to a model problem of three charged particles moving in one dimension (9). Chris was able to compute the cross section and electron energy distributions for this model using a semiclassical Green's function for a system with two non-separable coordinates. He found that the Wannier threshold law did indeed obtain but that there were some small departures from Wannier's theory in the energy distribution of the two electrons. These results encouraged Chris to extend the analytical-numerial methods to the real problem of three-particle motion in three dimensions. It was at this point that my close interaction with Chris on this subject began. My own work emphasized the time-dependent propagator for three particles $(13,14)$ and Chris was very much interested in the connections between the two representations.

At about this time Chris organized a workshop at the Santa-Barbara Institute for Theoretical Physics on the subject of time-dependent phenomena. It was my good fortune to spend three weeks at this workshop where Chris revealed a talent that he had kept well hidden, namely, he was an excellent scientific administrator! His open attitude and penetrating intellect were just the right combination to stimulate enthusiasm for the subject of the workshop and to direct it along fruitful lines. One direction is represented by cross sections for ionization of atomic hydrogen by incident protons, since timedependent methods are the normal way to study ion-atom collisions. At the same time, the probabilty for ionization at threshold goes according to the general "Wannier" theory of Feagin (7). Ab. initio calculations by Ovchinnikov and Solov'ev (15) using methods appropriate for heavy ion impact give results that relate closely to the Wannier threshold law. Of particular importance is their analysis of the top-of-barrier mechansim for ionization which they treated quantitatively for impact energies well above threshold. Extension of this theory to the threshold region provides an alternative means of building a quantitataive theory of fragmentation reactions at threshold. Ovchinnikov and I have combined these methods with the time-dependent propagator theory to obtain a complete theory for the ionization of $H$ atoms by proton impact at threshold (16). This short contribution outlines our approach.

\section{GENERAL THEORY}

A fragmentation process is charactarized as one where some coordinate $R$ which measures the overall size of the system, becomes infinite. The generic

\section{DISCLAIMER}

This report was prepared as an account of work sponsored by an agency of the United States This report was prepared as an account of work sponsored by an agency thereof, nor any of their employees, makes any warranty, express or implied, or assumes any legal liability or responsibility for the accuracy, completeness, or usefulness of any information, apparatus, product, or process disclosed, or represents that its use would not infringe privately owned rights. Reference herein to any specific commercial product, process, or service by trade name, trademark, manufacturer, or otherwise does not necessarily constitute or imply its endorsement, recommendation, or favoring by the United States Government or any agency thereof. The views and opinions of authors expressed herein do not necessarily state or reflect those of the United States Government or any agency thereof. 
Schrödinger equation for such a system has the form

$$
-\frac{1}{2 M} \frac{d^{2} \Psi}{d R^{2}}+\mathcal{H}(R, x) \Psi=E \Psi
$$

where the set of all variables other than $R$ are denoted by $x$. It is important that the variables $x$ are dimensionless. This is immediately the case when $R$ represents the hyperradius (1), as is conventional for breakup of an atom into two electrons and a positively charged core. For ion-atom interactions $R$ usually represents the internuclear distance while the remaining coordinates include the radial coordinate of the electron. This latter coordiante has dimensions, but Solov'ev (17) introduced scaled variables $\mathrm{q}=\mathrm{r} / R$, where $\mathrm{r}$ is the electron coordinate relative to the center of mass of the two nuclei, to obtain the appropriate form of $\mathcal{H}$ with dimensionless variables.

Eq.(2.1) is normally integrated along the real axis to connect the solution at one point $R=R_{i}$ with the solution at another point $R=R_{f}$, but the solutions can be connected by integrating along any path in the complex R-plane. We use this flexibility to find a path along which good approximations are available. To find this path, adiabatic energy eigenvalues $\varepsilon_{n}(R)$ for complex $R$ are computed;

$$
\mathcal{H}(R ; x) \phi_{n}(R ; x)=\varepsilon_{n}(R) \phi_{n}(R ; x) .
$$

Following Demkov (18), we consider a function $\varepsilon(R)$ which is single-valued on a multisheeted Riemann surface. On the real axis the single function $\varepsilon(R)$ takes on different values $\varepsilon_{n}(R)$ depending upon which sheet the variable $R$ is located. For our purposes an equivalent alternative function $n(R)=$ $1 / \sqrt{-\varepsilon(R)}$ is more useful. Fig.(1) shows a portion of the Riemann surface for this function computed using the program of Ovchinnikov and Solov'ev (15) for $H_{2}^{+}$. The surface is a plot of the real part of $n(R)$ vs the complex variable $\sqrt{R}$. The essential features of this surface are the branch points where the various sheets are connected. The locus of branch points separates complex $R$ space into two regions. In the region near the real axis the energy eigenvalues have a Rydberg structure charactaristic of the separated atom $R \rightarrow \infty$ or united atom $R \rightarrow 0$ limits. The function $n(R)$ interpolates smoothly between these two limits. On the other side of the locus of branch points $\varepsilon(R)$ has a harmonic oscillator structure. This stucture emerges because the potential $V(x, y, z)$ of an electron in the field of two positively charged particles has a saddle point where $V(x, y, z)$ is quadratic in all directions, i. e. the potential is that of an harmonic oscillator. In directions parallel to the internuclear axis, the oscillator is inverted, and the motion is unstable for real $R$. For complex $R$, however, the spring constants are complex and the motion in all directions is stable, i. e. the eigenfunctions $\varphi_{n}(R ; x, y, z)$ are localized near the top of the potential barrier between the two positively charged nuclei. This harmonic oscillator structure is exploited to compute an asympotic expression $\varepsilon_{T}(R)$ for the energy eigenvalues in the harmonic oscillator region. We find 


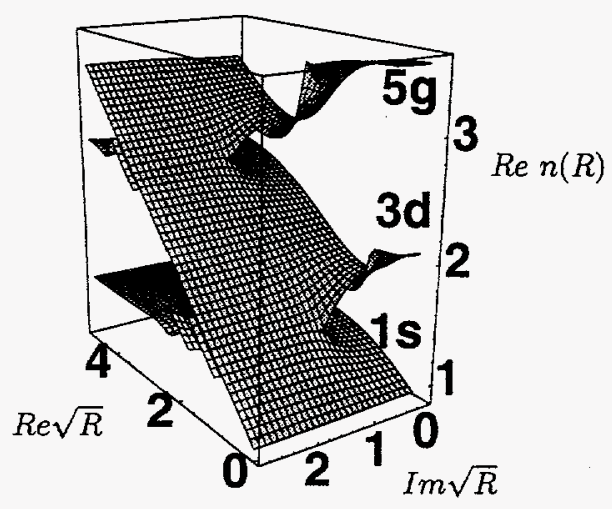

FIG. 1. Plot of $n(R)=1 / \sqrt{-\epsilon(R)}$ vs $\sqrt{R}$. "Back" view of the surface showing a sloping flat region to the left of an infinite series of branch points. The first two branch points of this series where the $1 s \sigma-3 d \sigma-5 g \sigma$ sheets join are seen in this view.

$\epsilon_{T}(R)=-\frac{C_{0}}{R}-\left(\frac{C_{1}^{\prime}+i \sqrt{C_{1}} / 2}{R^{3 / 2}}+\frac{C_{2}^{\prime}+i C_{2}}{R^{2}}+\frac{C_{3}^{\prime}+i C_{3}}{R^{5 / 2}}\right)+\mathcal{O}\left(R^{-3}\right)$

where, for $\mathrm{H}_{2}^{+}$the constants are

$$
\begin{aligned}
& C_{0}=3, \quad C_{1}=32, \quad C_{1}^{\prime}=-4, \\
& C_{2}=-3 \sqrt{2}, \quad C_{2}^{\prime}=3 / 2, \quad C_{3}=39 \sqrt{2} / 16, \quad C_{3}^{\prime}=9 / 16 .
\end{aligned}
$$

The probability for ionization is obtained by integrating the Schrodinger equation approximately in the one channel WKB approximation for motion in $R$. If this is done on the real axis the system remains in the initial channel, and the elastic scattering phase shift is obtained. If the integration contour circles a branch point connecting a surface $i$ with a surface $f$, the electronic wavefunction $\varphi(R ; \mathbf{q})$ starts out as $\varphi_{i}(R ; \mathbf{q})$ at $R_{i}$ on the real axis and ends up as $\varphi_{f}(R ; \mathbf{q})$ at $R_{f}$. The transition probability for the transition $i \rightarrow f$ is just given by

$$
P_{f i}=\left|\exp \left[\int_{c} K(R) d R\right]\right|^{2}
$$

where $K^{2}(R)=2 M[E-\varepsilon(R)]$ and $c$ indicates that the integral is taken along a contour in the complex plane connecting $R_{i}$ and $R_{f}$. In general, one must sum over all different contours that lead to the transition in question (19). For ionization, a contour that starts on the real axis at some small value of $R$, goes around the first top-of-barrrier branch point and to infinity on the 


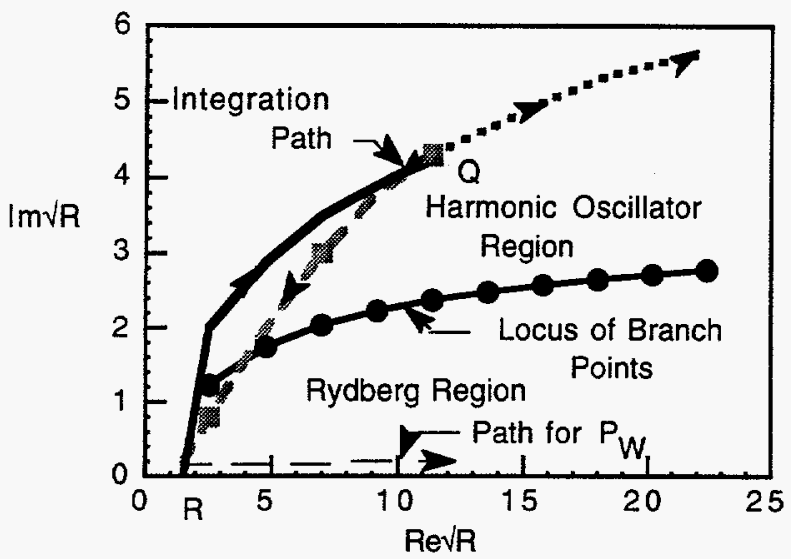

FIG. 2. Plot of the locus of branch points in the complex $\sqrt{R}$ plane. The smooth curve joining the points separates the plane into a region where the eigevalue spectrum is Rydberg-like and a region where it is harmonic oscillator-like. The integration path is also shown. From $R_{i}$ to $\mathrm{Q}$, the exact $\varepsilon(R)$ is used. From $\mathrm{Q}$ to $\infty$ the harmonic oscillator approximation is employed. Since this approximation corresonds to an $\varepsilon(R)$ without branch points, the contour can be deformed to run from $\mathrm{Q}$ back to $R_{i}$ and then to $\infty$ along the real axis. Integration along the real axis determines $P_{W}(E)$.

flat surface seen in Fig.(1) gives the probability for ionization via the topof-barrier mechansim. The path of integration around the branch points is shown in Fig.(2). At the point $\mathrm{Q}$, where $|R| \approx 200 \mathrm{au}$, the exact $\varepsilon(R)$ is well approximated by the asymptotic expression $\varepsilon_{T}(R)$. Now this asymptotic expression has no branch points, thus to compute the integral of this function we may distort the path so that it returns to $R_{i}$ and goes to infinity along the real axis. This gives the factored form for the transition probability

$$
P(E)=P_{0}(E) P_{W}(E)
$$

where

$$
P_{0}(E)=\left|\exp \left[\int_{i}^{Q}\left[K(R)-K_{T}(R)\right] d R\right]\right|^{2}
$$

and

$$
P_{W}(E)=\left|\exp \left[\int_{i}^{\infty} K_{T}(R) d R\right]\right|^{2}
$$

The function $P_{0}(E)$ is analytic in $\mathrm{E}$ so that the integral can be expanded in powers of $\mathrm{E}$. We find

$$
P_{0}(E)=\exp [-(0.94-0.6 E)]
$$


to lowest order in E. In contrast, $P_{W}(E)$ is not analytic in $E$ but the integrals can be done exactly. To lowest order in E, Eq.(2.8) together with the approximation

$$
K_{T}(R) \approx K_{0}(R)+\varepsilon_{T}(R) / v(R)
$$

where

$$
K_{0}(R)^{2}=2 M\left[E-C_{0} / R\right]=M^{2} v(R)^{2}
$$

gives

$$
P_{W}(E)=E^{\zeta_{a d}} \exp [-\sqrt{3 E}-(1 / 6) E \log E]
$$

with

$$
\zeta_{a d}=69.7 .
$$

The cross section is proportional to $P(E)$ thus the factor $P_{W}(E)$ gives the Wannier threshold law for the ionization of atomic hydrogen by proton impact. In addition we also extract the non-analytic $\sqrt{E}$ and $E \log E$ terms which represent anharmonic corrections to the Wannier threshold law. These terms have been missed in the now standard theories of the cross sections for breakup of three charged particles. It should also be noted that the $\sqrt{E}$ term is absent for the model of three particles confined to a line. This latter model has been solved essentially exactly by Chris (9) and classically by Rost and Heller (21).

The one-channel adiabatic calculation of ionization has been compared with measurements of the $H^{+}+H \rightarrow H^{+}+H^{+}+e$ by Pieksma and Ovchinnikov (20) and by Pieksma et al. (22) at velocities well above the threshold region. These workers find good agreement between theory and experiment thus this method is well established when $1 / M<<1$. In addition the approximate index $\zeta_{a d}$ that we obtain is in error by only terms of the order of magnitude of $1 / M$. Alternatively, for the ionization of hydrogen by electron impact, $\zeta_{a d}$ is in error by an unacceptable factor of $23 \%$. This indicates that wave function propagation in the harmonic oscillator region is not adequately described by the one-channel adiabatic theory. It is at this point that we connect with the work of Chris who introduced more general semiclassical methods. In our own work, semiclassical methods identify $d R / v(R)$ with a new variable $d t=d R / v(R)$. In the harmonic oscillator region where $R$ and $t$ are complex, Eq.(2.1) becomes a Schrödinger equation for a harmonic oscillator with time-dependent spring constants $k(t)$ and masses $m(t)$. Feynman's propagator $K_{F}\left(t, \mathbf{q} ; t^{\prime}, \mathbf{q}^{\prime}\right)$ for such time dependent oscillators is known exactly thus the lowest order solution is also known exactly. In essence the Schrödinger equation is integrated from $Q$ to infinity using the harmonic oscillator approximation rather than the one-channel WKB approxmation. As for this latter solution, one may change the path of integration to go from $\mathrm{Q}$ to $R_{i}$ and then 
from $R_{i}$ to infinity. The integration from $R_{i}$ to infinity along the real axis gives the threshold law with the correct Wannier index $\zeta_{W}$ rather than $\zeta_{a d}$.

There is a simple connection between $\zeta_{a d}$ and $\zeta_{W}$ which emerges upon examining the form of $K_{F}\left(t, \mathbf{q} ; t^{\prime}, \mathbf{q}^{\prime}\right)$. Along the real axis $K_{F}\left(t, \mathbf{q} ; t^{\prime}, \mathbf{q}^{\prime}\right)$ just equals a phase factor times a normalization constant. The normalization constant has the form

$$
N(t)=\exp \left[\int_{i}^{f} u\left(t^{\prime}\right) d t^{\prime}\right]
$$

where $u(t)$ is a known function. Since $d t=d R / v(R)$ we define a diabatic energy $\varepsilon_{d}(R, E)$ according to

$$
\varepsilon_{d}(R, E)=u[t(R, E)]
$$

This diabatic energy is expanded in powers of $E$ and only the first term is retained. This gives an expression $\varepsilon_{d}(R, 0)$ that is proportional to $R^{-3 / 2}$ just as is the adiabatic value; only the proportionality constants $C_{1}^{\prime}$ and $C_{1}$ are changed. This result was actually anticipated earlier by Rau (2). Substitution of these diabatic values of the constants into Eqs.(2.8) an expression for $P_{W}(E)$ that differs from Eq.(2.12) only through the replacement of $\zeta_{a d}$ by the correct Wannier index $\zeta_{W}$. In this way we see that propagation through the harmonic oscillator region using Feynman's propagator yields the correct Wannier threshold law. Higher order terms in $\varepsilon_{T}(R)$ still give the non-analytic $\sqrt{E}$ term in the cross section as obtained in the one-channel approximation.

The above results pertain to the Wannier threshold for ionization by proton impact. For these processes the method gives absolute cross sections as well as an analytic form for the cross section which includes the usual Wannier power law $E^{\zeta W}$ and the non-anlaytic $\sqrt{E}$ and $E \log E$ terms that represent the effects of anharmonic corrections in the asymptotic region. At present there are no data on the Wannier threshold law for proton impact, thus to test the non-analytic terms we consider ionization by electron impact. For this system, a complete theory requires the computation of $\epsilon_{T}(R)$ in hyperspherical coordinates. This has not been done yet, however, as Feagin shows, one can describe the factor $P_{W}(E)$ which comes from the harmonic oscillator region using the Born-Oppenheimer representation where $R$ is the inter-electron coordinate. For electron impact one finds that the function $P_{W}(E)$ is given by

$$
P_{W}(E)=E^{1.1269} \exp [1.1269(-\sqrt{3 E / 2})]
$$

The $\sqrt{E}$ correction that we find is unexpected and has not been obtained by other means. To test this result we have fitted the data of McGowan and Clark (23) over the extend energy range from 0 to $8 \mathrm{eV}$ above threshold. Here it is necessary to include a linear term with a coefficient fitted to the data. The coefficient is found to equal 0.9 . With this fitting we obtain good 


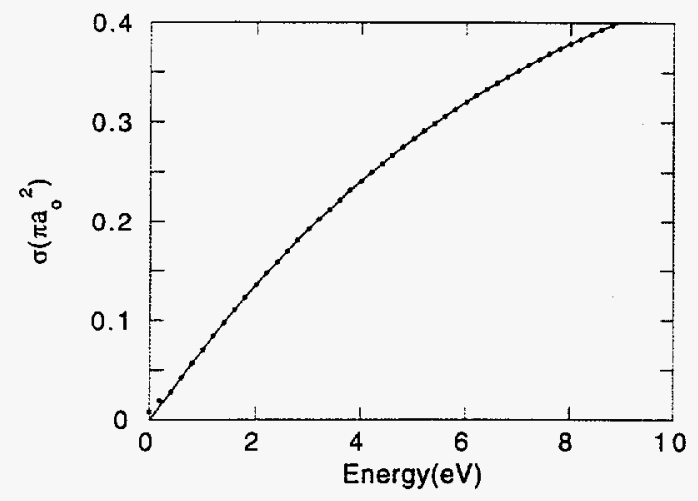

FIG. 3. Comparison of the fitted function $P(E)$ with the experimental data (dots) of Ref. (23)

agreement over the entire energy range as seen in Fig.(3). A recent classical calculation using the model with two electrons and the nucleus confined to a line also agrees well with the data. For this latter model the $\sqrt{E}$ term is absent in the quantal theory whereas we find that it represents the most important correction to Wannier's threshold law for the real system. We are not able to explain this contradiction at present. In this connection it should be noted that if the square root term is omitted but the linear term retained, the data cannot be fitted over the $8 \mathrm{eV}$ energy range.

For ionization by proton impact the present theory has no adjustable parameters. A similarly complete theory for electron impact requires the calculation of adiabatic hyperspherical eigenvalues for complex $R$. This is a difficult task, but, by using the basis spline programs developed by Chris and coworkers (24), it has proved possible to calculate just such eigenvalues. It seems that Chis is with us in spirit as we continue exploring this problem which held such great facination for him.

\section{ACKNOWLEDGMENTS}

Support for collaboration with the Ioffe Physical Technical Institute, St. Petersberg, Russia is provided by the National Science Foundation under grant no. PHY-9213953. This research is sponsored by the Division of Chemical Sciences, U.S. Department of Energy, under Contract No. DE-AC05-84OR21400. Thanks are also due to the Santa Barbara Center for Theoretical Physics for providing and enviorenment where these ideas were discussed with colleagues from all parts of the US and especially to Chis Bottcher his excellent direction of the workshop on time-dependent phenomena. 


\section{REFERENCES}

* Permanent Address: Ioffe Physical Techincal Institute, St. Petersburg, Russia.

1. G. H. Wannier, Phys. Rev. 90, 817 (1953).

2. A. R. P. Rau, Phys. Rev. A 4, 207 (1971).

3. R. Peterkop, J. Phys. B 4, 513 (1971).

4. H. Klar and W. Schlecht, J. Phys. B 9, 1699 (1976).

5. U. Fano, Physics Today 1, 1 (1976).

6. U. Fano, Rep. Prog. Phys. 46, 97 (1983).

7. James M. Feagin, J. Phys. B 17, 2433 (1984).

8. A. K. Kazansky and V. N. Ostrovsky, J. Phys. B 25, 2121 (1992).

9. C. Bottcher, Adv. in At. and Mol. Phys., 25, 303 (1989).

10. M. J. Alguard, V. W. Hughes, M. S. Lubell and P. F. Wainwright, Phy. Rev. Lett. 39, 334 (1977).

11. C. H. Greene and A. R. P. Rau, Phy. Rev. Lett., 48, 533 (1982).

12. D. S. Crothers, J. Phys. B 19, 463 (1986).

13. D. Jakubassa-Amundsen and J. Macek, J. Phys. A 22, $4151 \mathrm{~F}(1989)$.

14. J. H. Macek, Phys. Rev. A 41, 1361 (1990).

15. S.Y. Ovchinnikov and E.A. Solov'ev, Sov. Phys.-JETP, 64, 280 (1986); S. Y. Ovchinnikov and E. A. Solov'ev, Comments on Atomic and Molecular Physics XXII, 69 (1988).

16. J. H. Macek and S. Y. Ovchinnikov, Phys. Rev. A 49. R4273 (1994).

17. E. A. Solov'ev and S. I. Vinitsky, J. Phys. B 18, L557 (1985).

18. Yu. N. Demkov, Proceedings of invited talks of the V ICPEAC, Leningrad, USSR, July (1967)(Published by Joint Institute for Laboratory Astrophysics, Boulder, Colorado, 1968), p 186.

19. L.D.Landau and E.M.Lifshitz. Quantum Mechanics:Non-Relativistic Theory, 2nd. ed., (Pergamon Press, Oxford, England 1965).

20. M. Pieksma and S. Y. Ovchinnikov, J. Phys. B 24, 2699 (1991).

21. J. M. Rost and E. Heller, Phys. Rev. Lett. ? , (1994).

22. M. Pieksma, S. Y. Ovchinnikov, J. van Eck, W. B. Westerfeld, and A. Niehaus, Phys. Rev. Lett., 73, 48 (1994).

23. J. W. McGowan and E. M. Clarke, Phys. Rev. 167, 43 (1968)

24. J. Wells, V. E. Oberacker,A. S. Umar, C. Bottcher, M. R. Strayer, J. S. Wu, and G. Plunien, Phys. Rev. A, 6296 (1992). 